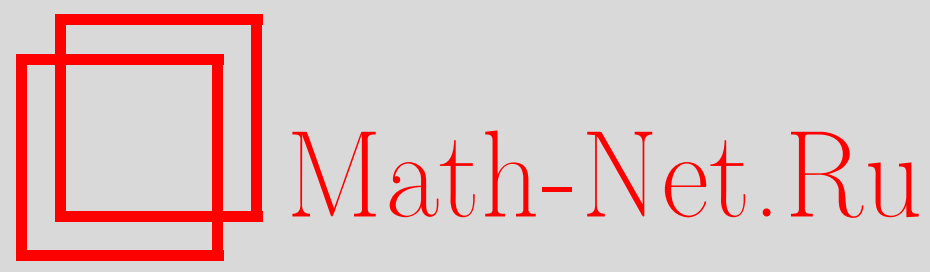

В. Б. Васильев, Волновая факторизация эллиптических символов, Матем. заметки, 2000, том 68, выпуск 5, 653667

DOI: https://doi.org/10.4213/mzm987

Использование Общероссийского математического портала Math-Net.Ru подразумевает, что вы прочитали и согласны с пользовательским соглашением http://www . mathnet.ru/rus/agreement

Параметры загрузки:

IP : 54.80 .73 .141

26 апреля 2023 г., 16:06:10

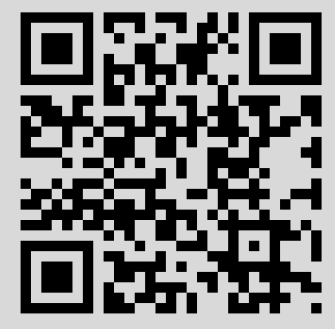


УДК 517.95

\title{
ВОЛНОВАЯ ФАКТОРИЗАЦИЯ ЭЛЛИПТИЧЕСКИХ СИМВОЛОВ
}

\section{В.Б. Васильев}

\begin{abstract}
Введено понятие волновой факторизации символа эллиптического псевдодифференциального оператора и показано применение волновой факторизации к исследованию псевдодифференциальных уравнений в конусах.
\end{abstract}

Библиография: 13 названий.

0. В теории краевых задач для эллиптических псевдодифференциальных уравнений, разработанной М.И. Вишиком, Г.И. Эскиным [1], [2], ключевую роль сьграла факторизация символа псевдодифференциального оператора по одной из переменных при фиксированных остальных. В зависимости от так называемого индекса факторизации приходилось рассматривать различные краевые задачи, отражающие особенности возникшей ситуации. Рассматривались также и алгебры краевых задач [3], [4], с помощью которых можно было охватить все ситуации. При изучении псевдодифференциального уравнения в конусе (угле) автором настоящей работы было обнаружено, что аналогичную роль будет играть “волновая" факторизация, связанная с выходом в комплексную область по всем переменным сразу. Такая факторизация позволяет использовать многомерньй вариант метода Винера-Хопфа и получить для псевдодифференциального уравнения в угле такую же картину разрешимости, какая имела место в случае полупространства. Такой подход применим и к некоторым классам дифференциальных операторов и до сих пор не использовался [5]. Цель настоящей работы - убедиться в том, что запас эллиптических символов, допускающих волновую факторизацию, достаточно богат и продемонстрировать в одном случае "работу" волновой факторизации.

1. Пусть $A(\xi), \xi \in \mathbb{R}^{m},-$ символ псевдодифференциального оператора $A$, удовлетворяющий условию

$$
c_{1} \leqslant\left|A(\xi)(1+|\xi|)^{-\alpha}\right| \leqslant c_{2},
$$

где $c_{1}, c_{2}$ - положительные постоянные. Число $\alpha \in \mathbb{R}$ назовем порядком псевдодифференциального оператора $A$. Очевидно, что символ $A(\xi)$ эллиптический, т. е. $A(\xi) \neq 0$ $\forall \xi \in \mathbb{R}$.

Обозначим через $C_{+}^{a}$ конус в пространстве $\mathbb{R}^{m}$ вида

$$
\begin{aligned}
& C_{+}^{a}=\left\{\xi \in \mathbb{R}^{m}:\left|\xi^{\prime}\right|<a \xi_{m}, \xi^{\prime}=\left(\xi_{1}, \ldots, \xi_{m-1}\right), a>0\right\}, \\
& \stackrel{C}{C}_{+}^{a}=\left\{\xi \in \mathbb{R}^{m}: a\left|\xi^{\prime}\right|<\xi_{m}\right\} .
\end{aligned}
$$

Радиальной трубчатой областью над конусом $C_{+}^{a}$ назьвается $[6]$ множество из $\mathbb{C}^{m}$ вида $\mathbb{R}^{m}+i C_{+}^{a} \equiv T\left(C_{+}^{a}\right)$.

Работа выполнена при финансовой поддержке Российского фонда фундаментальных исследований, грант № 96-01-01698. 
ОПРЕДЕЛЕНИЕ 1. Волновой факторизаиией символа $A(\xi)$ относительно конуса $C_{+}^{a}$ назьвается его представление в виде

$$
A(\xi)=A_{\neq}(\xi) A_{=}(\xi),
$$

где сомножители $A_{\neq}(\xi), A_{=}(\xi)$ должны обладать свойствами:

1) $A_{\neq}(\xi), A_{=}(\xi)$ определены всюду на $\mathbb{R}^{m}$, кроме, быть может, множества точек $\left\{\xi \in \mathbb{R}^{m}:\left|\xi^{\prime}\right|^{2}=a^{2} \xi_{m}^{2}\right\}$

2) $A_{\neq}(\xi), A_{=}(\xi)$ допускают аналитическое продолжение в $T\left(\stackrel{*}{C_{+}^{a}}\right), T\left(\stackrel{*}{C}_{-}^{a}\right)$ соответственно, удовлетворяющее оценкам

$$
\begin{aligned}
& \left|A_{\neq}^{ \pm 1}(\xi+i \tau)\right| \leqslant c_{1}(1+|\xi|+|\tau|)^{ \pm \varkappa}, \\
& \left|A_{=}^{ \pm 1}(\xi-i \tau)\right| \leqslant c_{2}(1+|\xi|+|\tau|)^{ \pm(\alpha-\varkappa)} \forall \tau \in \stackrel{C}{C}_{+}^{*},
\end{aligned}
$$

где $\stackrel{*}{C_{-}^{a}}-$ конус, противоположньцй конусу $\stackrel{*}{C}_{+}^{a}: \stackrel{*}{C}_{-}^{a}=-\stackrel{*}{C}_{+}^{a}$.

Число назовем индексом волновой факторизации, а саму волновую факторизацию для сокращения записи будем именовать $w$-факторизацией.

Для сравнения мы приведем определение факторизации "по Вишику-Эскину", которая применялась для изучения псевдодифференциальных уравнений в полупространстве $\left\{\xi \in \mathbb{R}^{m}: \xi_{m}>0\right\}$.

Пусть $C_{\alpha}^{\infty}, \alpha \in \mathbb{R},-$ класс эллиптических символов $A(\xi)$, которые бесконечно дифференцируемы при $\xi \neq 0$ и представляют собой однородные функции порядка $\alpha$, т.е.

$$
A(t \xi)=t^{\alpha} A(\xi) \quad \forall t>0 .
$$

ОПРЕДЕЛЕНИЕ 2 [2]. Однородной факторизацией эллиптического символа $A(\xi) \in$ $C_{\alpha}^{\infty}$ по переменной $\xi_{m}$ называется представление $A\left(\xi^{\prime}, \xi_{m}\right), \xi^{\prime}=\left(\xi_{1}, \ldots, \xi_{m-1}\right)$, в виде

$$
A\left(\xi^{\prime}, \xi_{m}\right)=A_{+}\left(\xi^{\prime}, \xi_{m}\right) A_{-}\left(\xi^{\prime}, \xi_{m}\right)
$$

где $A_{+}\left(\xi^{\prime}, \xi_{m}\right)$ и $A_{-}\left(\xi^{\prime}, \xi_{m}\right)$ удовлетворяют следующим условиям:

a) $A_{+}\left(\xi^{\prime}, \xi_{m}\right)$ при $\xi^{\prime} \neq 0$ допускает аналитическое продолжение по $\xi_{m}$ в верхнюю полуплоскость $\tau>0$;

б) $A_{+}\left(\xi^{\prime}, \xi_{m}+i \tau\right)$ непрерьвна по совокупности переменных $\left(\xi^{\prime}, \xi_{m}, \tau\right)$ при $\tau \geqslant 0$, $\left|\xi^{\prime}\right|+\left|\xi_{m}\right|+\tau>0$

в) $A_{+}\left(\xi^{\prime}, \xi_{m}+i \tau\right)-$ однородная функция порядка $\lambda$ :

$$
A_{+}\left(t \xi^{\prime}, t\left(\xi_{m}+i \tau\right)\right)=t^{\lambda} A_{+}\left(\xi^{\prime}, \xi_{m}+i \tau\right) \quad \forall t>0 ;
$$

г) $A_{+}\left(\xi^{\prime}, \xi_{m}+i \tau\right) \neq 0$ при $\tau \geqslant 0,\left|\xi^{\prime}\right|+\left|\xi_{m}\right|+\tau>0$.

Аналогично, функция $A_{-}\left(\xi^{\prime}, \xi_{m}\right)$ допускает при $\xi^{\prime} \neq 0$ аналитическое продолжение по $\xi_{m}$ в полуплоскость $\tau<0$, непрерьвна и отлична от нуля при $\tau \leqslant 0,\left|\xi^{\prime}\right|+\left|\xi_{m}\right|+|\tau|>0$ и однородна порядка $\alpha-\lambda$.

В [2] кроме этого требовалось вьполнение условия

$$
A_{+}(0 ;+1)=1 \text {. }
$$

Оказалось, что справедлива следующая 
Tеорема 1 [2]. Пусть $A(\xi) \in C_{\alpha}^{\infty}$ - әллиптический символ. Тогда $A(\xi)$ допускает единственную при условии нормировки (2) однородную факторизачию по переменной $\xi_{m}$. Коши.

Эта факторизация была построена в [2] конструктивно с помощью интеграла типа

Для волновой факторизации у нас нет аналога теоремы 1 (кстати, можно обратить внимание, что в определении $w$-факторизации отсутствует условие типа б) - требование непрерывности). Однако мы покажем, что по любому эллиптическому символу класса $C_{\alpha}^{\infty}$ можно построить другой эллиптический символ, который уже допускает $w$-факторизацию.

2. Пусть

$$
A=-\frac{\partial^{2}}{\partial x_{1}^{2}}-\cdots-\frac{\partial^{2}}{\partial x_{m}^{2}}+k^{2}, \quad k \in \mathbb{R} \backslash\{0\} .
$$

Тогда $A(\xi)=\xi_{1}^{2}+\xi_{2}^{2}+\cdots+\xi_{m-1}^{2}+\xi_{m}^{2}+k^{2} \equiv|\xi|^{2}+k^{2}$.

Лемма 1. Символ $A(\xi)$ допускает $w$-факторизачию с индексом 1 вида

$$
\begin{aligned}
A(\xi)=( & \left.\sqrt{a^{2}+1} \xi_{m}+\sqrt{a^{2} \xi_{m}^{2}-\xi_{1}^{2}-\cdots-\xi_{m-1}^{2}-k^{2}}\right) \\
& \times\left(\sqrt{a^{2}+1} \xi_{m}-\sqrt{a^{2} \xi_{m}^{2}-\xi_{1}^{2}-\cdots-\xi_{m-1}^{2}-k^{2}}\right),
\end{aligned}
$$

əде под $\sqrt{a^{2} \xi_{m}^{2}-\xi_{1}^{2}-\cdots-\xi_{m-1}^{2}-k^{2}}$ понимается граничное значение аналитической в $T\left(\stackrel{*}{C}_{+}^{a}\right)$ функиии $\sqrt{a^{2} z_{m}^{2}-z_{1}^{2}-\cdots-z_{m-1}^{2}-k^{2}}$.

ДокАЗАТЕЛЬСтво. Рассмотрим функцию

$$
\sqrt{a^{2}+1} z_{m}+\sqrt{a^{2} z_{m}^{2}-z_{1}^{2}-\cdots-z_{m-1}^{2}-k^{2}}
$$

как функцию $m$ комплексных переменных $z_{j}=\xi_{j}+i \eta_{j}, j=1,2, \ldots, m$.

Нетрудно убедиться в том, что функция $a^{2} z_{m}^{2}-z_{1}^{2}-\cdots-z_{m-1}^{2}-k^{2}$ не принимает неотрицательных значений при $z=\left(z_{1}, \ldots, z_{m}\right) \in T\left(\stackrel{*}{C}_{+}^{a}\right) \cup T\left(\stackrel{*}{C_{-}^{a}}\right)$ (в [6, лемма на с. 350] это доказано в случае $a \equiv 1, k \equiv 0$; обший случай рассматривается дословным повторением приведенных там рассуждений). Отсюда следует, что каждая из двух ветвей функции $W(z)=\sqrt{a^{2} z_{m}^{2}-z_{1}^{2}-\cdots-z_{m-1}^{2}-k^{2}}$ однозначна и аналитична в $T\left(\stackrel{*}{C}_{+}^{a}\right) \cup T\left(\stackrel{*}{C}_{-}^{a}\right) \equiv T\left(\stackrel{*}{C}^{a}\right)$.

Следуя [6], введем класс $H_{0}\left(\stackrel{*}{C}^{a}\right)$ функций $f(z)$, аналитических в радиальной трубчатой области $T\left(\stackrel{*}{C}^{a}\right)\left(\stackrel{*}{C}^{a} \equiv \stackrel{*}{C}_{+}^{a} \cup \stackrel{*}{C}_{-}^{a}\right)$ над конусом $\stackrel{*}{C}^{a}$ и таких, что для любого подконуса $\stackrel{*}{C_{1}^{a}}$, компактного в $\stackrel{*}{C}$, они удовлетворяют оценке

$$
|f(z)| \leqslant M\left(\stackrel{*}{C}_{1}^{a}\right)(1+|z|)^{\beta}\left(1+|y|^{-\alpha}\right), \quad z \in T\left(\stackrel{*}{C}_{1}^{a}\right),
$$

при некоторых $\alpha, \beta \geqslant 0$, независящих от $\stackrel{*}{C}_{1}^{a}, z=x+i y ; M\left(\stackrel{*}{C_{1}^{a}}\right)$ - постоянная, зависящая от подконуса $\stackrel{*}{C}_{1}^{a}$. 
Нетрудно заметить, что функция $W(z)$ удовлетворяет оценке (4) с $\alpha=0, \beta=1$ и универсальной постоянной $M$ (не зависящей от подконуса $\left.\stackrel{*}{C}_{1}^{a}\right)$. Таким образом, $W(z) \in$ $H_{0}\left(\stackrel{*}{C}^{a}\right)$ и, следовательно [6], имеет граничные значения в смысле распределений при $y \rightarrow 0, y \in \stackrel{*}{C}_{ \pm}^{a}$. Эти граничные значения являются локально интегрируемыми функциями и легко вычисляются. Поскольку граничные значения не зависят от того, по какому пути $y$ стремится к нулю, можно рассмотреть пределы вида

$$
\lim _{\varepsilon \rightarrow 0+} \sqrt{a^{2}\left(x_{m} \pm i \varepsilon\right)^{2}-x_{1}^{2}-\cdots-x_{m-1}^{2}-k^{2}}
$$

Выберем одну из ветвей функции $W(z)$, ту, которая отображает $T\left(\stackrel{*}{C}^{a}\right)$ на верхнюю полуплоскость. Граничные значения этой ветви будут иметь следующий вид:

$$
\begin{aligned}
& \sqrt{a^{2}\left(x_{m} \pm i 0\right)^{2}-x_{1}^{2}-\cdots-x_{m-1}^{2}-k^{2}} \\
& \quad=\left\{\begin{array}{lll} 
\pm \sqrt{a^{2} x_{m}^{2}-\left|x^{\prime}\right|^{2}-k^{2}}, & a^{2} x_{m}^{2}-\left|x^{\prime}\right|^{2}-k^{2}>0, & x_{m}>0 \\
\mp \sqrt{a^{2} x_{m}^{2}-\left|x^{\prime}\right|^{2}-k^{2}}, & a^{2} x_{m}^{2}-\left|x^{\prime}\right|^{2}-k^{2}>0, & x_{m}<0 \\
i \sqrt{\left|x^{\prime}\right|^{2}+k^{2}-a^{2} x_{m}^{2}}, & a^{2} x_{m}-\left|x^{\prime}\right|^{2}-k^{2}<0,
\end{array}\right.
\end{aligned}
$$

который получается, если аккуратно проследить за поведением модуля и аргумента функции $W\left(x_{1}, \ldots, x_{m-1}, x_{m} \pm i \varepsilon\right)$ при $\varepsilon \rightarrow 0$.

Далее отметим, что

$$
\sqrt{a^{2}\left(x_{m}+i 0\right)^{2}-\left|x^{\prime}\right|^{2}-k^{2}}=-\sqrt{\sqrt{a^{2}\left(x_{m}-i 0\right)^{2}-\left|x^{\prime}\right|^{2}-k^{2}}}
$$

где черта сверху обозначает комплексное сопряжение.

Возвращаясь к функции $\sqrt{a^{2}+1} z_{m}+W(z)$, заключаем, что она аналитична в $T\left({ }^{*} C_{+}^{a}\right)$, и, аналогично, функция $\sqrt{a^{2}+1} z_{m}+\overline{W(z)}$ аналитична в $T(\stackrel{*}{C} \underset{-}{a})$. Действительно, коль скоро $W(z) \in H_{0}\left(\stackrel{*}{C}_{+}^{a}\right)$, то по теореме 2 из [6, с. 280] для нее существует спектральная функция $g(\xi)$, обрашаюшаяся в нуль вне $C_{+}^{a}$ и такая, что

$$
W(z)=\int_{C_{+}^{a}} g(\xi) e^{i z \xi} d \xi, \quad z \in \stackrel{*}{C}_{+}^{a} .
$$

Нетрудно убедиться в том, что

$$
\begin{gathered}
\overline{W(z)}=\overline{\int_{C_{+}^{a}} g(\xi) e^{i z \xi} d \xi}=\int_{C_{-}^{a}} \overline{g(-\xi)} e^{i\left(\bar{z}_{1} \xi_{1}+\cdots+\bar{z}_{m} \xi_{m}\right)} d \xi \\
\stackrel{\text { обозн }}{\equiv} G\left(\bar{z}_{1}, \ldots, \bar{z}_{m}\right), \quad z=\left(z_{1}, \ldots, z_{m}\right) \in T\left(\stackrel{(}{C}_{+}^{a}\right) .
\end{gathered}
$$

Тогда, очевидно, $\bar{z}=\left(\bar{z}, \ldots, \bar{z}_{m}\right) \in T\left(\stackrel{*}{C}_{-}^{a}\right)$ и поскольку для функции $G(z), z \in T\left(\stackrel{*}{C}_{-}^{a}\right)$, имеется спектральная функция $\overline{g(-\xi)}$, обращающаяся в нуль вне $C_{-}^{a}$, то по той же теореме 2 из $[6$, с. 280$] G(z) \in H_{0}\left(\stackrel{*}{C}_{-}^{a}\right)$. 
Следовательно, можно записать

$$
\begin{aligned}
\xi_{m}^{2}+\left|\xi^{\prime}\right|^{2}+k^{2}= & \left(\sqrt{a^{2}+1} \xi_{m}+\sqrt{a^{2}\left(\xi_{m}+i 0\right)^{2}-\left|\xi^{\prime}\right|^{2}-k^{2}}\right) \\
& \times\left(\sqrt{a^{2}+1} \xi_{m}+\frac{\sqrt{a^{2}\left(\xi_{m}-i 0\right)^{2}-\left|\xi^{\prime}\right|^{2}-k^{2}}}{}\right)
\end{aligned}
$$

где каждый из сомножителей допускает аналитическое продолжение в $T\left(\stackrel{*}{C}_{+}^{a}\right), T\left(\stackrel{*}{C}_{-}^{a}\right)$ класса $H_{0}\left(\stackrel{*}{C_{+}^{a}}\right), H_{0}\left(\stackrel{*}{C_{-}^{a}}\right)$ соответственно.

С учетом связи между граничными значениями из $T\left(\stackrel{*}{C}_{+}^{a}\right)$ и $T\left(\stackrel{*}{C}_{-}^{a}\right)$ формула (3) (а вместе с ней и лемма 1) доказана.

Можно обобщить формулу (3), рассмотрев “степени” оператора Гельмгольца, т. е. операторы с символами

$$
\left(\xi_{m}^{2}+\left|\xi^{\prime}\right|^{2}+k^{2}\right)^{\alpha+i \beta}, \quad \alpha, \beta \in \mathbb{R}
$$

В этом случае $w$-факторизация будет выглядеть так:

$$
\begin{aligned}
\left(\xi_{m}^{2}+\left|\xi^{\prime}\right|^{2}+k^{2}\right)^{\alpha+i \beta}= & \left(\sqrt{a^{2}+1} \xi_{m}+\sqrt{a^{2} \xi_{m}^{2}-\left|\xi^{\prime}\right|^{2}-k^{2}}\right)^{\alpha+i \beta} \\
& \times\left(\sqrt{a^{2}+1} \xi_{m}-\sqrt{a^{2} \xi_{m}^{2}-\left|\xi^{\prime}\right|^{2}-k^{2}}\right)^{\alpha+i \beta} .
\end{aligned}
$$

Аналитичность сомножителей в $T\left(\stackrel{*}{C}_{+}^{a}\right), T\left(\stackrel{*}{C}_{-}^{a}\right)$ следует из того, что функция $\sqrt{a^{2}+1} z_{m}+W(z)$ не имеет нулей в $T\left(\stackrel{*}{C}_{+}^{a}\right)$, так как при $z \in T\left(\stackrel{*}{C_{+}^{a}}\right)$ имеем $\operatorname{Im} z_{m}>0$, $\operatorname{Im} W(z)>0$ и область значений этой функции односвязна.

ЗАмЕчАнИЕ. Если рассмотреть однородные символы класса $C_{\alpha}^{\infty}$ ( $\alpha$ в данном случае - порядок однородности) и в определении $w$-факторизации в условии 2) потребовать однородности степени $\varkappa$ у $A_{\neq}$и степени $\alpha-\varkappa$ у $A_{=}$, то можно говорить об однородной $w$-факторизации.

ПримеР. В формуле (5) положим $k=0$ и получим однородную $w$-факторизацию $|\xi|^{\alpha+i \beta}$ :

$$
\begin{aligned}
|\xi|^{\alpha+i \beta}= & \left(\sqrt{a^{2}+1} \xi_{m}+\sqrt{a^{2} \xi_{m}^{2}-\left|\xi^{\prime}\right|^{2}}\right)^{(\alpha+i \beta) / 2} \\
& \times\left(\sqrt{a^{2}+1} \xi_{m}-\sqrt{a^{2} \xi_{m}^{2}-\left|\xi^{\prime}\right|^{2}}\right)^{(\alpha+i \beta) / 2} .
\end{aligned}
$$

Наконец, если в формуле (6) положить $a \equiv 0$ (в этом случае конусы ${ }_{C}^{*} \pm$ “вырождаются" в полупространства $\left.\mathbb{R}_{ \pm}^{m}=\left\{\xi \in \mathbb{R}^{m}: \pm \xi_{m}>0\right\}\right)$, получится однородная факторизация по переменной $\xi_{m}$ в смысле определения 2:

$$
|\xi|^{\alpha+i \beta}=\left(\xi_{m}+i\left|\xi^{\prime}\right|\right)^{(\alpha+i \beta) / 2}\left(\xi_{m}-i\left|\xi^{\prime}\right|\right)^{(\alpha+i \beta) / 2},
$$

хорошо известная в теории Вишика-Эскина [2, с. 67]. 
3. Пусть теперь $\sigma(\xi)$ - эллиптический символ класса $C^{\infty}$ при $\xi \neq 0$ и однородный степени $\alpha$. По теореме 1 он допускает однородную факторизацию по переменной $\xi_{m}$ :

$$
\sigma\left(\xi^{\prime}, \xi_{m}\right)=\sigma_{+}\left(\xi^{\prime}, \xi_{m}\right) \sigma_{-}\left(\xi^{\prime}, \xi_{m}\right)
$$

Зафиксируем $\xi^{\prime}=\left(a_{1}, \ldots, a_{m-1}\right) \equiv a^{\prime}$ и рассмотрим

$$
\sigma_{+}\left(a^{\prime}, \xi_{m}\right) \sigma_{-}\left(a^{\prime}, \xi_{m}\right)
$$

В выражении (7) сделаем замену переменной $\xi_{m} \mapsto \sqrt{a^{2}+1} \xi_{m}+\sqrt{a^{2} \xi_{m}^{2}-\left|\xi^{\prime}\right|^{2}}$ для первого сомножителя и $\xi_{m} \mapsto \sqrt{a^{2}+1} \xi_{m}-\sqrt{a^{2} \xi_{m}^{2}-\left|\xi^{\prime}\right|^{2}}$ для второго сомножителя, приводя, таким образом, (7) к виду

$$
\sigma_{+}\left(a^{\prime}, \sqrt{a^{2}+1} \xi_{m}+\sqrt{a^{2} \xi_{m}^{2}-\left|\xi^{\prime}\right|^{2}}\right) \sigma_{-}\left(a^{\prime}, \sqrt{a^{2}+1} \xi_{m}-\sqrt{a^{2} \xi_{m}^{2}-\left|\xi^{\prime}\right|^{2}}\right) .
$$

Выражение (8) корректно, так как несмотря на то, что функции $\sqrt{a^{2}+1} \xi_{m} \pm$ $\sqrt{a^{2} \xi_{m}^{2}-\left|\xi^{\prime}\right|^{2}}$ принимают комплексные значения, эти значения расположены в верхней и нижней полуплоскостях, т. е. там, куда аналитически продолжаются (и, следовательно, где определены) функции $\sigma_{+}, \sigma_{-}$.

Обозначим через

$$
\begin{aligned}
& \widetilde{\sigma}\left(a^{\prime}, \xi\right) \\
& \quad=\sigma_{+}\left(a^{\prime}, \sqrt{a^{2}+1} \xi_{m}+\sqrt{a^{2} \xi_{m}-\left|\xi^{\prime}\right|^{2}}\right) \sigma_{-}\left(a^{\prime}, \sqrt{a^{2}+1} \xi_{m}-\sqrt{a^{2} \xi_{m}^{2}-\left|\xi^{\prime}\right|^{2}}\right)
\end{aligned}
$$

символ, зависящий от $(m-1)$-мерного параметра $a^{\prime}$. Формула (9) представляет собой $w$-факторизацию символа $\widetilde{\sigma}\left(a^{\prime}, \xi\right)$. Действительно, аналитичность функций

$$
\sigma_{+}\left(a^{\prime}, \sqrt{a^{2}+1} \xi_{m}+\sqrt{a^{2} \xi_{m}^{2}-\left|\xi^{\prime}\right|^{2}}\right), \quad \sigma_{-}\left(a^{\prime}, \sqrt{a^{2}+1} \xi_{m}-\sqrt{a^{2} \xi_{m}^{2}-\left|\xi^{\prime}\right|^{2}}\right)
$$

в $T\left(\stackrel{*}{C}_{+}^{a}\right), T(\stackrel{*}{C} \underset{-}{a})$ соответственно следует из того, что композиция аналитических функций является аналитической функцией, из аналитичности функций $\sigma_{+}, \sigma_{-}$в $\mathbb{C}_{+}, \mathbb{C}_{-}$ (где $\mathbb{C}_{+}, \mathbb{C}_{-}-$верхняя и нижняя комплексные полуплоскости) и из того, что функции (которые определены на разных множествах; см. доказательство леммы 1)

$$
\sqrt{a^{2}+1} z_{m}+\sqrt{a^{2} z_{m}^{2}-z_{1}^{2}-\cdots-z_{m-1}^{2}}, \quad \sqrt{a^{2}+1} z_{m}+\sqrt{\sqrt{a^{2} z_{m}^{2}-z_{1}^{2}-\cdots-z_{m-1}^{2}}}
$$

аналитичны как отображения $T\left(\stackrel{*}{C}_{ \pm}^{a}\right) \rightarrow \mathbb{C}_{ \pm}$соответственно.

Наконец, оценки, вьполнения которых требует условие 2) определения 1 , вытекают из однородности и непрерывности $\sigma_{+}, \sigma_{-}$при любом $a^{\prime} \neq 0$. Правда, получаемый при этой операции символ $\widetilde{\sigma}\left(a^{\prime}, \xi\right)$ не принадлежит, вообще говоря, классу $C_{\alpha}^{\infty}$, однако он удовлетворяет неравенству $\left(1_{\alpha}\right)$, и, следовательно, псевдодифференциальньй оператор с таким символом ограниченно действует из пространства $H_{s}\left(\mathbb{R}^{m}\right)$ в пространство $H_{s-\alpha}\left(\mathbb{R}^{m}\right)($ см. $[2$, лемма 4.4, c. 45$])$. 
4. В пш. 4, 5 мы рассмотрим специальные типы символов - символы многомерньх сингулярных интегральных операторов, при изучении которых также было использовано понятие $w$-факторизации [7].

Пусть $A(\xi)$ - однородньй степени 0 эллиптический символ класса $C^{\infty}\left(\mathbb{R}^{m} \backslash\{0\}\right)$ (и, таким образом, удовлетворяющий условию $\left.\left(1_{0}\right)\right)$. При фиксированном $\xi^{\prime}$ его можно представить в виде сходящегося ряда

$$
A(\xi)=\sum_{k=-\infty}^{\infty} c_{k}\left(\xi_{m}-i\left|\xi^{\prime}\right|\right)^{k}\left(\xi_{m}+i\left|\xi^{\prime}\right|\right)^{-k}
$$

так как система функций $\left\{\left(\xi_{m}-i\left|\xi^{\prime}\right|\right)^{k}\left(\xi_{m}+i\left|\xi^{\prime}\right|\right)^{-k}\right\}_{-\infty}^{+\infty}$ образует базис в пространстве $L_{2}(\mathbb{R})$. Предположим, что $A(\xi)$ допускает факторизацию по переменной $\xi_{m}$ (в смысле определения 2):

$$
A(\xi)=A_{+}(\xi) A_{-}(\xi)
$$

в классе сходящихся рядов

$$
\begin{aligned}
& A_{+}(\xi)=\sum_{k=0}^{\infty} a_{k}\left(\xi_{m}-i\left|\xi^{\prime}\right|\right)^{k}\left(\xi_{m}+i\left|\xi^{\prime}\right|\right)^{-k}, \\
& A_{-}(\xi)=\sum_{k=-\infty}^{-1} b_{k}\left(\xi_{m}-i\left|\xi^{\prime}\right|\right)^{k}\left(\xi_{m}+i\left|\xi^{\prime}\right|\right)^{-k} .
\end{aligned}
$$

Очевидно, что $A(\xi), A_{+}(\xi), A_{-}(\xi)$ - однородные степени 0 функции и, стало быть, индекс факторизации по переменной $\xi_{m}$ равен 0 . Наше предположение равносильно тому, что $A(0, \ldots, 0,+1)=A(0, \ldots, 0,-1)$ и приращение аргумента функции $A(\xi)$ вдоль какой-нибудь большой полуокружности единичной сферы $S^{m-1}$, соединяющей точки $(0, \ldots, 0,-1)$ и $(0, \ldots, 0,+1)$ (в случае $m=2$ правой и левой полуокружностей), равно нулю. Действительно, в этом случае по теореме 1 факторизация по $\xi_{m}$ существует, и $A_{+}, A_{-}$как непрерывные функции разлагаются (при фиксированной $\xi^{\prime}$ ) в сходящиеся ряды $Ф$ урье по положительньп и отрицательным степеням $\left(\xi_{m}-i\left|\xi^{\prime}\right|\right)^{k}\left(\xi_{m}+i\left|\xi^{\prime}\right|\right)^{-k}$ соответственно.

Перемножая (10) и (11), получим совокупность соотношений

$$
\begin{aligned}
c_{0} & =a_{1} b_{-1}+a_{2} b_{-2}+\cdots+a_{n} b_{-n}+\cdots, \\
c_{1} & =a_{2} b_{-1}+a_{3} b_{-2}+\cdots+a_{n} b_{-(n-1)}+\cdots, \\
c_{-1} & =a_{1} b_{-2}+a_{2} b_{-3}+\cdots+a_{n} b_{-(n+1)}+\cdots,
\end{aligned}
$$

причем каждый числовой ряд бесконечной системы (12) является сходящимся.

Рассмотрим теперь совокупность $\mathscr{A}$ однородных степени 0 функций, представимых сходящимися рядами

$$
\stackrel{\sqcap}{A}(\xi)=\sum_{k=-\infty}^{\infty} c_{k}\left(\sqrt{a^{2}+1} \xi_{m}-\sqrt{a^{2} \xi_{m}^{2}-\left|\xi^{\prime}\right|^{2}}\right)^{k}\left(\sqrt{a^{2}+1} \xi_{m}+\sqrt{a^{2} \xi_{m}^{2}-\left|\xi^{\prime}\right|^{2}}\right)^{-k} .
$$

Обозначим

$$
\begin{aligned}
& A_{\neq}(\xi)=\sum_{k=0}^{\infty} a_{k}\left(\sqrt{a^{2}+1} \xi_{m}-\sqrt{a^{2} \xi_{m}^{2}-\left|\xi^{\prime}\right|^{2}}\right)^{k}\left(\sqrt{a^{2}+1} \xi_{m}+\sqrt{a^{2} \xi_{m}^{2}-\left|\xi^{\prime}\right|^{2}}\right)^{-k} \\
& A_{=}(\xi)=\sum_{k=-\infty}^{-1} b_{k}\left(\sqrt{a^{2}+1} \xi_{m}-\sqrt{a^{2} \xi_{m}^{2}-\left|\xi^{\prime}\right|^{2}}\right)^{k}\left(\sqrt{a^{2}+1} \xi_{m}+\sqrt{a^{2} \xi_{m}^{2}-\left|\xi^{\prime}\right|^{2}}\right)^{-k}
\end{aligned}
$$


Теорема 2. Пусть $\stackrel{П}{A}(\xi) \in \mathscr{A}$. Если существует последовательности чисел $\left\{a_{k}\right\}_{k=0}^{\infty},\left\{b_{k}\right\}_{k=-\infty}^{-1}$ такие, что сходятся ряды (13) и (14) и вылолняются соотношения (12), то $A(\xi)$ допускает однородную $w$-факторизацию с индексом, равным нулю.

Казалось бы, теорему 2 применить практически невозможно, однако в случае конечных сумм это сделать относительно просто.

ПРИМЕР. Пусть $a \equiv 1$,

$$
\stackrel{\sqcap}{A}(\xi)=3 l^{-1}(\xi)+7+2 l(\xi)
$$

где $l(\xi)=\left(\sqrt{2} \xi_{m}-\sqrt{\xi_{m}^{2}-\left|\xi^{\prime}\right|^{2}}\right)\left(\sqrt{2} \xi_{m}+\sqrt{\xi_{m}^{2}-\left|\xi^{\prime}\right|^{2}}\right)^{-1}$.

Символ $\stackrel{П}{A}(\xi)$ эллиптический, так как $\stackrel{\sqcap}{A}(\xi) \neq 0 \quad \forall \xi \neq 0$. Действительно, $\stackrel{\sqcap}{A}(\xi)=0$, только если $l(\xi)=-3,-1 / 2$, однако функция $l(\xi)$ не принимает этих значений. Проверим это, например, для -3 . Если $l(\xi)=-3$, то

$$
\sqrt{2} \xi_{m}-\sqrt{\xi_{m}^{2}-\left|\xi^{\prime}\right|^{2}}=-3 \sqrt{2} \xi_{m}-3 \sqrt{\xi_{m}^{2}-\left|\xi^{\prime}\right|^{2}}, \quad 28 \xi_{m}^{2}+4\left|\xi^{\prime}\right|^{2}=0 .
$$

Последнее возможно лишь тогда, когда $\xi_{m}=0,\left|\xi^{\prime}\right|=0$, т. е. $\xi=0$.

Аналогично проверяется значение $-1 / 2$.

Ищем $A_{\neq}(\xi), A_{=}(\xi)$ в виде

$$
A_{\neq}(\xi)=a_{0}+a_{1} l(\xi), \quad A_{=}(\xi)=b_{0}+b_{-1} l^{-1}(\xi) .
$$

Условия (12) дают систему

$$
\left\{\begin{array}{l}
a_{0} b_{0}+a_{1} b_{-1}=7 \\
a_{1} b_{0}=2 \\
a_{0} b_{-1}=3
\end{array}\right.
$$

откуда получаем $b_{0}=2 a_{1}^{-1}, b_{-1}=3 a_{0}^{-1}$.

Подставляя это в первое уравнение, имеем

$$
2 a_{0} a_{1}^{-1}+3 a_{1} a_{0}^{-1}=7 .
$$

Обозначая $a_{0} a_{1}^{-1}=k$, получаем уравнение

$$
2 k+3 k^{-1}=7
$$

которое имеет два корня $k_{1}=1 / 2, k_{2}=3$, в результате чего появляются два варианта $w$-факторизации

$$
\stackrel{\Pi}{A}(\xi)=(1+2 l(\xi))\left(1+3 l^{-1}(\xi)\right), \quad \stackrel{\sqcap}{A}(\xi)=(3+l(\xi))\left(2+l^{-1}(\xi)\right)
$$

(стоит обратить внимание, что $w$-факторизация получается с точностью до произвольной постоянной), однако первьй вариант не подходит, потому что функция $1+2 l(\xi)$ имеет нули в $T\left(\stackrel{*}{C}_{+}^{1}\right)$, а функция $1+3 l^{-1}(\xi)$ - в $T\left(\stackrel{*}{C}_{-}^{1}\right)$. Рассмотрим, например, уравнение

$$
1+2 l(z)=0, \quad z \in T\left(C_{+}^{1}\right)
$$


(в случае $a=1, \stackrel{*}{C}_{+}^{1}=C_{+}^{1}$ ). Имеем

$$
\begin{aligned}
0 & =1+2 l(z)=1+2 \frac{\sqrt{2} z_{m}-\sqrt{z_{m}^{2}-z_{1}^{2}-\cdots-z_{m-1}^{2}}}{\sqrt{2} z_{m}+\sqrt{z_{m}^{2}-z_{1}^{2}-\cdots-z_{m-1}^{2}}} \\
& =\frac{3 \sqrt{2} z_{m}-\sqrt{z_{m}^{2}-z_{1}^{2}-\cdots-z_{m-1}^{2}}}{\sqrt{2} z_{m}+\sqrt{z_{m}^{2}-z_{1}^{2}-\cdots-z_{m-1}^{2}}} \\
& =\frac{3 \sqrt{2} z_{m}-\operatorname{Re} \sqrt{z_{m}^{2}-z_{1}^{2}-\cdots-z_{m-1}^{2}}-i \operatorname{Im} \sqrt{z_{m}^{2}-z_{1}^{2}-\cdots-z_{m-1}^{2}}}{\sqrt{2} z_{m}+\sqrt{z_{m}^{2}-z_{1}^{2}-\cdots-z_{m-1}^{2}}} .
\end{aligned}
$$

Поскольку знаменатель последней дроби не имеет нулей в $T\left(C_{+}^{1}\right)$, ишем точки из $T\left(C_{+}^{1}\right)$, в которых

$$
3 \sqrt{2} z_{m}-\operatorname{Re} \sqrt{z_{m}^{2}-z_{1}^{2}-\cdots-z_{m-1}^{2}}-i \operatorname{Im} \sqrt{z_{m}^{2}-z_{1}^{2}-\cdots-z_{m-1}^{2}}=0
$$

Положим $x_{m}=y_{1}=\cdots=y_{m-1}=0$. Уравнение (15) примет вид

$$
3 \sqrt{2} i y_{m}-\operatorname{Re} \sqrt{-y_{m}^{2}-x_{1}^{2}-\cdots-x_{m-1}^{2}}-i \operatorname{Im} \sqrt{-y_{m}^{2}-x_{1}^{2}-\cdots-x_{m-1}^{2}}=0 .
$$

Из (16) следует, что $3 \sqrt{2} i y_{m}-i \sqrt{y_{m}^{2}+x_{1}^{2}+\cdots+x_{m-1}^{2}}=0$, откуда получаем

$$
y_{m}=\frac{1}{\sqrt{17}}\left|x^{\prime}\right|, \quad y_{m}>0 .
$$

Таким образом, мы показали, что точки $z=\left(z_{1}, \ldots, z_{m}\right)$, координаты которых удовлетворяют условию (17) (они, очевидно, принадлежат $T\left(C_{+}^{1}\right)$ ), являются решениями уравнения $1+2 l(z)=0$. Конечно, аналогично можно рассмотреть и более сложные примеры $w$-факторизаций функций вида

$$
\stackrel{\Pi}{A}(\xi)=\sum_{k=-n}^{n} c_{k} l^{k}(\xi) .
$$

5. В этом пункте опять $A(\xi)$ - однородньй степени 0 эллиптический символ класса $C^{\infty}\left(\mathbb{R}^{m} \backslash\{0\}\right)$. Обозначим через $F$ преобразование Фурье в смысле обобщенных функций [2], supp $A(\xi)$ - носитель (обобщенный) функции $A(\xi)$.

TeOpema 3. Пусть $\operatorname{supp} F^{-1}(\ln A(\xi)) \subset C_{+}^{a} \cup C_{-}^{a}$. Тогда $A(\xi)$ допускает однородную ш-факторизачию с нулевым индексом. 
ДоКАЗАТЕЛЬСТВо. Обозначим

$$
K(x)=F_{\xi \rightarrow x}^{-1}(\ln A(\xi))
$$

Хорошо известно, что $K(x)$ представляет собой сингулярное ядро Михлина-Кальдерона-Зигмунда класса $C^{\infty}\left(\mathbb{R}^{m} \backslash\{0\}\right)[8]$ - функцию, однородную степени $-m$. Запишем

$$
K=\chi_{+} K+\chi_{-} K
$$

где $\chi_{ \pm}-$характеристическая функция множества $C_{ \pm}^{a}$.

$\chi_{+} K$ можно рассматривать как обобщенную функцию медленного роста с носителем в $C_{+}^{a}$. Тогда ее преобразование Фурье-Лапласа принадлежит классу $H\left(\stackrel{*}{C_{+}^{a}}\right)$ функций $f$, аналитических в $\stackrel{*}{C}_{+}^{a}$ и допускающих оценку (см. [6, с. 280 , теорема 2])

$$
|f(z)| \leqslant M\left(C^{\prime}\right)(1+|z|)^{\beta}\left(1+|y|^{-\alpha}\right), \quad z \in T\left(C^{\prime}\right)
$$

при некоторых $\alpha \geqslant 0, \beta \geqslant 0$, не зависящих от $C^{\prime} ; C^{\prime}-$ подконус, компактньй в $\stackrel{*}{C}_{+}^{a}$, $M\left(C^{\prime}\right)$ - постоянная, зависяшая лишь от подконуса $C^{\prime}[6]$.

Убедимся теперь в том, что граничные значения преобразования Фурье-Лапласа функции $\chi_{+} K$ ограничены. Действительно, эти граничные значения совпадают с преобразованием $\Phi$ урье в смысле распределений функции $\chi_{+} K$ (обобщенная функция v. p. $K(x)$ корректно определена, например, в [9]), что равносильно преобразованию Фурье в смысле главного значения [8]. Хорошо известно, что оператор Михлина-Кальдерона-Зигмунда с ядром $\chi_{+} K$ будет ограниченным оператором, действующим в пространстве $L_{2}\left(\mathbb{R}^{m}\right)[8]$, и, следовательно, его символ - преобразование Фурье в смыслеглавного значения ядра - обязан быть ограниченным, так как оператор Михлина-Кальдерона-Зигмунда с ядром $\chi_{+} K$ в пространстве $L_{2}\left(\mathbb{R}^{m}\right)$ унитарно эквивалентен оператору умножения на символ.

Теперь ограниченность преобразования Фурье-Лапласа функции $\chi_{+} K$ следует из ограниченности его граничных значений (см. [10, теорема на с. 179]). Очевидно, аналогичное утверждение справедливо в отношении функции $\chi-K$.

Вернемся к равенству (18). Так как supp $K \subset C_{+}^{a} \cup C_{-}^{a}$, то представление $K$ в виде (18) правомерно и, более того, однозначно (исключая тривиальньй случай $K(x)=$ $c \delta(x)$, при котором $A(\xi)=$ const). Переходя в (18) к преобразованию Фурье и потенцируя, получим $A(\xi)=A_{\neq}(\xi) A_{=}(\xi)$, где $A_{\neq}(\xi)=\exp F\left(\chi_{+} K\right), A_{=}(\xi)=\exp F\left(\chi_{-} K\right)$, и $A_{\neq}, A_{=}$обладают всеми свойствами, наличия которых требует определение 1.

6. В этом пункте выясним вопрос о единственности $w$-факторизации.

ТЕОРема 4. Если әллиптический символ $A(\xi)$ допускает $w$-факторизачию относительно конуса $C_{+}^{a}$, то она единственна с точностью до мультипликативной постоянной.

ДокАЗАТЕЛЬСтво. Пусть имеется $w$-факторизация $A(\xi)=A_{\neq}(\xi) A_{=}(\xi)$ с индексом $\varkappa$ и еше одна $w$-факторизация $A(\xi)=B_{\neq}(\xi) B_{=}(\xi)$ с индексом $\gamma$. Запишем $A_{\neq}(\xi) \times$ $B_{\neq}^{-1}(\xi)=A_{=}^{-1}(\xi) B_{=}(\xi)$. Тогда $A_{\neq}(z) B_{\neq}^{-1}(z)$ аналитична в $T\left(\stackrel{*}{C_{+}^{a}}\right)$ с оценкой

$$
\left|A_{\neq}(z) B_{\neq}^{-1}(z)\right| \leqslant c(1+|z|)^{\varkappa-\gamma}
$$


а $A_{=}^{-1}(z) B_{=}(z)$ аналитична в $T\left(\stackrel{*}{C}_{-}^{a}\right)$ с оценкой

$$
\left|A_{=}^{-1}(z) B_{=}(z)\right| \leqslant c(1+|z|)^{\varkappa-\alpha+\alpha-\gamma}=c(1+|z|)^{\varkappa-\gamma},
$$

т. е. $A_{\neq} B_{\neq}^{-1} \in H\left(\stackrel{*}{C_{+}^{a}}\right), A_{=}^{-1} B_{=} \in H\left(\stackrel{*}{C}_{-}^{a}\right)$.

Тогда по теореме из $[6$, с. 290$]$ функция

$$
f(z)= \begin{cases}A_{\neq}(z) B_{\neq}^{-1}(z), & z \in T\left(\stackrel{*}{C}_{+}^{a}\right), \\ A_{=}^{-1}(z) B_{=}(z), & z \in T\left(\stackrel{*}{C}_{-}^{a}\right),\end{cases}
$$

принадлежит классу $H(C)$, где $C$ - вьпуклая оболочка $\stackrel{*}{C}_{+}^{a} \cup \stackrel{*}{C} a$ совпадает с $\mathbb{R}^{m}$. Более того, эта функция $f(z)$ имеет степенной порядок на бесконечности и не имеет нулей в $T\left(\stackrel{*}{C}_{+}^{a}\right) \cup T\left(\stackrel{*}{C}_{-}^{a}\right)$. По теореме Лиувилля [6] $f(z)$ должна быть полиномом, однако любой полином будет иметь нули либо в $\mathbb{R}^{m}$, либо в $T\left(\stackrel{*}{C}_{+}^{a}\right) \cup T\left(\stackrel{*}{C}_{-}^{a}\right)$. Остается единственная возможность $f(z)=$ const и, следовательно,

$$
A_{\neq}(\xi)=c B_{\neq}(\xi), \quad A_{=}(\xi)=c^{-1} B_{=}(\xi) .
$$

Понятно, что если задать “условие нормировки” типа (2), то $w$-факторизация символа $A(\xi)$ будет единственной.

7. В этом пункте мы покажем, как $w$-факторизация может быть применена к изучению псевдодифференциальных уравнений в конусе. Всюду ниже $m=2$.

Рассмотрим псевдодифференциальное уравнение в $C_{+}^{a}$ вида

$$
P A u_{+}=f
$$

где $u_{+} \in H_{s}\left(C_{+}^{a}\right)$, т.е. $u_{+} \in H_{s}\left(\mathbb{R}^{2}\right), \operatorname{supp} u_{+} \subset \bar{C}_{+}^{a}, f$ задана в $C_{+}^{a}, P$ - оператор сужения на $C_{+}^{a}$, т. е.

$$
(P u)(x)=u(x), \quad x \in C_{+}^{a}, \quad(P u)(x)=0, \quad x \notin \bar{C}_{+}^{a},
$$

$A$ - псевдодифференциальный оператор с символом $A(\xi) \in C_{\alpha}^{\infty}$.

Пусть $S\left(\mathbb{R}^{2}\right)$ - класс Шварца бесконечно дифференцируемых функций на плоскости $\mathbb{R}^{2}$, убьваюших при $|x|=\sqrt{x_{1}^{2}+x_{2}^{2}} \rightarrow \infty$ быстрее любой отрицательной степени $|x|$ вместе со всеми своими производньпи. Для $f \in S\left(\mathbb{R}^{2}\right)$ определим оператор $G_{2}$ :

$$
\left(G_{2} f\right)(\xi)=\lim _{\tau \rightarrow 0+} \int_{\mathbb{R}^{2}} \frac{f\left(\eta_{1}, \eta_{2}\right) d \eta_{1} d \eta_{2}}{\left(\xi_{1}-\eta_{1}\right)^{2}-a^{2}\left(\xi_{2}-\eta_{2}+i \tau\right)^{2}}
$$

Обозначим через $F$ оператор преобразования Фурье:

$$
(F f)\left(\xi_{1}, \xi_{2}\right)=\int_{\mathbb{R}^{2}} e^{i\left(x_{1} \xi_{1}+x_{2} \xi_{2}\right)} f\left(x_{1}, x_{2}\right) d x_{1} d x_{2} \equiv \tilde{f}(\xi)
$$

Обозначим через $\widetilde{H}_{s}\left(\mathbb{R}^{2}\right), \widetilde{H}_{s}\left(C_{+}^{a}\right), \widetilde{H}_{s}\left(\mathbb{R}^{2} \backslash \bar{C}_{+}^{a}\right)$ Фурье-образы пространств $H_{s}\left(\mathbb{R}^{2}\right)$, $H_{s}\left(C_{+}^{a}\right), H_{s}\left(\mathbb{R}^{2} \backslash \bar{C}_{+}^{a}\right)$ соответственно. 
Напомним, что пространство $H_{s}\left(\mathbb{R}^{2}\right), s \in \mathbb{R}$, состоит [2] из обобщенных функций $u$, преобразование Фурье которых является локально интегрируемой по Лебегу функцией $\widetilde{u}(\xi)$ такой, что

$$
\|u\|_{s}^{2}=\int_{\mathbb{R}^{2}}|\widetilde{u}(\xi)|^{2}(1+|\xi|)^{2 s} d \xi<+\infty .
$$

Псевдодифференциальньй оператор $A$ с символом $A(\xi)$ (вообще говоря, это распределение из $\left.S^{\prime}\left(\mathbb{R}^{2}\right)\right)$ на функциях $u \in S\left(\mathbb{R}^{2}\right)$ определяется формулой

$$
(A u)(x)=F_{\xi \rightarrow x}^{-1}(A(\xi) \cdot \widetilde{u}(\xi)), \quad u \in S\left(\mathbb{R}^{2}\right),
$$

где $F^{-1}$ понимается в смысле распределений.

Напомним также [2], что $S\left(\mathbb{R}^{2}\right)$ плотно в $H_{s}\left(\mathbb{R}^{2}\right) \forall s \in \mathbb{R}$ и что псевдодифференциальньй оператор $A$ с символом $A(\xi) \in C_{\alpha}^{\infty}$ - ограниченньй оператор, действующий из пространства $H_{s}\left(\mathbb{R}^{2}\right)$ в пространство $H_{s-\alpha}\left(\mathbb{R}^{2}\right)$.

TеОрема 5. Пусть $|s|<1 / 2$. Тогда оператор $G_{2}$ - ограниченный проектор пространства $\widetilde{H}_{s}\left(\mathbb{R}^{2}\right)$ на подпространство $\widetilde{H}_{s}\left(C_{+}^{a}\right)$, и любая функция $\widetilde{f} \in \widetilde{H}_{s}\left(\mathbb{R}^{2}\right)$ однозначно представима в виде

$$
\widetilde{f}=\tilde{f}_{+}+\tilde{f}_{-}
$$

əде $\tilde{f}_{+} \in \widetilde{H}_{s}\left(C_{+}^{a}\right), \tilde{f}_{-} \in \widetilde{H}_{s}\left(\mathbb{R}^{2} \backslash \bar{C}_{+}^{a}\right)$, причем $\tilde{f}_{+}=G_{2} \tilde{f}, \tilde{f}_{-}=\left(I-G_{2}\right) \tilde{f}$.

ДокАЗАтЕльство. Ограниченность оператора $G_{2}$ в пространстве $\widetilde{H}_{s}\left(\mathbb{R}^{2}\right)$, очевидно, эквивалентна ограниченности оператора

$$
\left(G_{2}^{0} f\right)(x)=\lim _{\tau \rightarrow 0+} \int_{\mathbb{R}^{2}} \frac{f(y)(1+|y|)^{s} d y}{(1+|x|)^{s}\left(\left(x_{1}-y_{1}\right)^{2}-a^{2}\left(x_{2}-y_{2}+i \tau\right)^{2}\right)}
$$

в пространстве $L_{2}\left(\mathbb{R}^{2}\right)$ квадратично интегрируемых функций. Оператор $G_{2}$ можно записать в виде

$$
\left(G_{2} f\right)(x)=\frac{1}{2 a} \lim _{\tau \rightarrow 0+} \int_{-\infty}^{+\infty} \int_{-\infty}^{+\infty} \frac{f\left(t_{1}, t_{2}\right) d t_{1} d t_{2}}{\left(x_{1}-t_{1}-a i \tau\right)\left(x_{2}-t_{2}+a i \tau\right)}
$$

сделав замену переменных

$$
\xi_{1}-a \xi_{2}=x_{1}, \quad \xi_{1}+a \xi_{2}=x_{2}, \quad \eta_{1}-a \eta_{2}=t_{1}, \quad \eta_{1}+a \eta_{2}=t_{2} .
$$

Далее, воспользовавшись формулами Сохоцкого [11], получаем представление

$$
\left(G_{2} f\right)(x)=\frac{\pi^{2}}{2 a} f(x)+\frac{\pi i}{2 a}\left(S_{1} f\right)(x)-\frac{\pi i}{2 a}\left(S_{2} f\right)(x)+\frac{1}{2 a}\left(S_{12} f\right)(x),
$$

где $S_{12}=S_{1} S_{2}$

$$
\left(S_{1} f\right)(x)=\text { v.p. } \int_{-\infty}^{+\infty} \frac{f\left(y_{1}, x_{2}\right) d y_{1}}{y_{1}-x_{1}}, \quad\left(S_{2} f\right)(x)=\text { v.p. } \int_{-\infty}^{+\infty} \frac{f\left(x_{1}, y_{2}\right) d y_{2}}{y_{2}-x_{2}} .
$$

Отметим, что $1+|\xi| \sim 1+|x|, 1+|\eta| \sim 1+|y|$, т. е. отношение рассматриваемых величин ограничено сверху и снизу положительньми постоянньми. Наконец, воспользуемся 
ограниченностью операторов $S_{1}, S_{2}$ в пространстве $L_{2}\left(\mathbb{R}^{2}\right)$ с весом $(1+|x|)^{s}($ см. [2, лемма 5.3]). Таким образом, $G_{2}$ - ограниченный оператор в пространстве $\widetilde{H}_{s}\left(\mathbb{R}^{2}\right)$.

Обозначим через $\theta$ оператор умножения на характеристическую функцию множества $C_{+}^{a}$. Тот факт, что $G_{2}$ - проектор на подпространство $\widetilde{H}_{s}\left(C_{+}^{a}\right)$, следует из свойства преобразования $\Phi$ урье

$$
F(\theta \cdot u)=2 a G_{2} F u, \quad u \in S\left(\mathbb{R}^{2}\right) .
$$

Действительно, рассмотрим интеграл

$$
\int_{\mathbb{R}^{2}} e^{i x \xi} \chi_{+}(x) u(x) e^{-x_{2} \tau} d x,
$$

где $\chi_{+}(x)$ - характеристическая функция конуса $C_{+}^{a}$. Так как функции $\chi_{+}(x) e^{-x_{2} \tau}$ и $u(x)$ абсолютно интегрируемы, к (21) применима теорема о свертке. Найдем преобразование Фурье функции $\chi_{+}(x) e^{-x_{2} \tau}$. Пусть $\tau>0$. Тогда

$$
\begin{aligned}
\int_{\mathbb{R}^{2}} & e^{i x \xi} \chi_{+}(x) e^{-x_{2} \tau} d x=\int_{C_{+}^{a}} e^{i x_{1} \xi_{1}} e^{i x_{2}\left(\xi_{2}+i \tau\right)} d x_{1} d x_{2} \\
& =\int_{-\infty}^{+\infty}\left(\int_{a\left|x_{1}\right|}^{+\infty} e^{i x_{2}\left(\xi_{2}+i \tau\right)} d x_{2}\right) e^{i x_{1} \xi_{1}} d x_{1} \\
& =-\frac{1}{i\left(\xi_{2}+i \tau\right)} \int_{-\infty}^{+\infty} e^{i a\left|x_{1}\right|\left(\xi_{2}+i \tau\right)} e^{i x_{1} \xi_{1}} d x_{1}=\frac{2 a}{\xi_{1}^{2}-a^{2}\left(\xi_{2}+i \tau\right)^{2}} .
\end{aligned}
$$

Применение теоремы о свертке к интегралу (21) с последуюшим предельным переходом при $\tau \rightarrow 0$ дает формулу (20).

Ясно, что если $G_{2}-$ проектор на подпространство $\widetilde{H}_{s}\left(C_{+}^{a}\right)$, то $I-G_{2}-$ проектор на подпространство $\widetilde{H}_{s}\left(\mathbb{R}^{2} \backslash \bar{C}_{+}^{a}\right)$.

В силу ограниченности оператора $G_{2}$ в $\widetilde{H}_{s}\left(\mathbb{R}^{2}\right)$

$$
\tilde{f}=G_{2} \tilde{f}+\left(I-G_{2}\right) \tilde{f} \equiv \tilde{f}_{+}+\tilde{f}_{-},
$$

причем $G_{2} \tilde{f} \in \widetilde{H}_{s}\left(C_{+}^{a}\right),\left(I-G_{2}\right) \tilde{f} \in \widetilde{H}_{s}\left(\mathbb{R}^{2} \backslash \bar{C}_{+}^{a}\right)$.

Убедимся в единственности представления (22). Для этого достаточно показать, что равенство

$$
\tilde{f}_{+}+\tilde{f}_{-}=0
$$

где $\widetilde{f}_{+} \in \widetilde{H}_{s}\left(C_{+}^{a}\right), \tilde{f}_{-} \in \widetilde{H}_{s}\left(\mathbb{R}^{2} \backslash \bar{C}_{+}^{a}\right)$, возможно только в том случае, когда $\tilde{f}_{+}=\tilde{f}_{-}=0$.

С учетом свойств оператора $G_{2}$ имеем

$$
G_{2} \tilde{f}_{+}=\tilde{f}_{+}, \quad G_{2} \tilde{f}_{-}=0 \quad \text { и } \quad\left(I-G_{2}\right) \tilde{f}_{+}=0, \quad\left(I-G_{2}\right) \tilde{f}_{-}=\tilde{f}_{-} .
$$

Действуя на обе части равенства $(23)$ операторами $G_{2}$ и $I-G_{2}$, получаем $\tilde{f}_{+}=0$, $\tilde{f}_{-}=0$.

Вернемся к уравнению (19). Правую часть $f$ будем рассматривать в пространстве $H_{s-\alpha}^{\prime}\left(C_{+}^{a}\right)$ обобщенных функций $S^{\prime}\left(C_{+}^{a}\right)$ с носителем в $\bar{C}_{+}^{a}$, допускающих продолжение $l f$ на $\mathbb{R}^{2}$, принадлежащее $H_{s-\alpha}\left(\mathbb{R}^{2}\right)$. Норму в $H_{s-\alpha}^{\prime}\left(C_{+}^{a}\right)$ зададим равенством [2]

$$
\|f\|_{s-\alpha}^{+}=\inf \|l f\|_{s-\alpha}
$$

где нижняя грань берется по всем продолжениям $f$, принадлежащим $H_{s-\alpha}\left(\mathbb{R}^{2}\right)$. 
Теорема 6. Пусть символ $A(\xi) \in C_{\alpha}^{\infty}$ оператора $A$ допускает $w$-факторизацию относительно конуса $C_{+}^{a}$ с индексом . Если $|\varkappa-s|<1 / 2$, то уравнение (19) для любой правой части $f \in H_{s-\alpha}^{\prime}\left(C_{+}^{a}\right)$ имеет единственное решение $u_{+} \in H_{s}\left(C_{+}^{a}\right)$, преобразование Фурье которого записывается в виде

$$
\widetilde{u}_{+}=A_{\neq}^{-1} G_{2} A_{=}^{-1} \widetilde{l f}
$$

где lf - произвольное продолэсение $f$ в $H_{s-\alpha}\left(\mathbb{R}^{2}\right)$.

Имеет место априорная оченка

$$
\left\|u_{+}\right\|_{s} \leqslant c\|f\|_{s-\alpha}^{+} .
$$

ДокАЗАТЕЛЬСТво. Пусть $l f-$ произвольное продолжение $f \in H_{s-\alpha}^{\prime}\left(C_{+}^{a}\right)$ на $H_{s-\alpha}\left(\mathbb{R}^{2}\right)$. Обозначим

$$
u_{-}=l f-A u_{+} .
$$

Применяя к (24) преобразование Фурье с учетом $w$-факторизации, получим

$$
A_{\neq}(\xi) \widetilde{u}_{+}(\xi)+A_{=}^{-1}(\xi) \widetilde{u}_{-}(\xi)=A_{=}^{-1}(\xi) \widetilde{l f}(\xi) .
$$

Так как символы $A_{\neq}^{ \pm 1}(\xi), A_{=}^{ \pm 1}(\xi)$ удовлетворяют оценкам

$$
\left|A_{\neq}^{ \pm 1}(\xi)\right| \leqslant c(1+|\xi|)^{ \pm \varkappa}, \quad\left|A_{=}^{ \pm 1}(\xi)\right| \leqslant c(1+|\xi|)^{ \pm \alpha \mp \varkappa}
$$

то псевдодифференциальные операторы $A_{\neq}, A_{=}^{-1}$ ограниченно действуют: $A_{\neq}: H_{s}\left(C_{+}^{a}\right)$ $\rightarrow H_{s-\varkappa}\left(\mathbb{R}^{2}\right), A_{=}^{-1}: H_{s-\alpha}\left(\mathbb{R}^{2} \backslash \bar{C}_{+}^{a}\right) \rightarrow H_{s-\varkappa}\left(\mathbb{R}^{2}\right)$ (см. [2, лемма 4.4]) и, следовательно, $A_{\neq}(\xi) \widetilde{u}_{+}(\xi), A_{=}^{-1}(\xi) \widetilde{u}_{-}(\xi), A_{=}^{-1}(\xi) \widetilde{l f}(\xi)$ принадлежат пространству $\widetilde{H}_{s-\varkappa}\left(\mathbb{R}^{2}\right)$. Отметим, что $u_{-} \in H_{s-\alpha}\left(\mathbb{R}^{2}\right)$, так как оператор $A$ ограниченно действует из пространства $H_{s}\left(\mathbb{R}^{2}\right)$ в $H_{s-\alpha}\left(\mathbb{R}^{2}\right)$, поскольку $A(\xi) \in C_{\alpha}^{\infty}$.

Покажем, что $A_{\neq}(\xi) \widetilde{u}_{+}(\xi) \in \widetilde{H}_{s-\varkappa}\left(C_{+}^{a}\right), A_{=}^{-1}(\xi) \widetilde{u}_{-}(\xi) \in \widetilde{H}_{s-\varkappa}\left(\mathbb{R}^{2} \backslash \bar{C}_{+}^{a}\right)$.

Из оценок $A_{\neq}^{ \pm 1}(\xi+i \tau), A_{=}^{ \pm 1}(\xi-i \tau)$ при $\tau \in \stackrel{*}{C}_{+}^{a}$ вытекает, что они входят в $H_{0}\left(\stackrel{*}{C_{ \pm}^{a}}\right)$ соответственно, и, следовательно, их спектральные функции $\omega_{+}^{ \pm 1}(x), \omega_{-}^{ \pm 1}(x)$ (преобразование Фурье-Лапласа $A_{\neq 1}^{ \pm 1}(\xi+i \tau), A_{=}^{ \pm 1}(\xi-i \tau)$ в смысле распределений) существуют и имеют носители в $C_{ \pm}^{a}[6]$.

Возьмем аппроксимацию функций $u_{+}$и $u_{-}$функциями $u_{+}^{(\varepsilon)}, u_{-}^{(\varepsilon)}$, бесконечно дифференцируемьми и финитными в $\bar{C}_{+}^{a}$ и $\mathbb{R}^{2} \backslash C_{+}^{a}$ соответственно (плотность таких функций в шкале пространств $H_{s}\left(C_{+}^{a}\right)$ и $H_{s}\left(\mathbb{R}^{2} \backslash \bar{C}_{+}^{a}\right)$ можно доказать методами работы [2] путем незначительных технических усложнений). Тогда определены свертки $\omega_{+} * u_{+}^{(\varepsilon)}$, $\omega_{-}^{-1} * u_{-}^{(\varepsilon)}$ обобщенных и основных функций $\left(\omega_{+}(y), \overline{u_{+}^{(\varepsilon)}(x-y)}\right),\left(\omega_{-}^{-1}(y), \overline{u_{-}^{(\varepsilon)}(x-y)}\right)$, где $\overline{u_{ \pm}^{(\varepsilon)}(x-y)}$ рассматривается как функция $y$ ( $x$ фиксировано), т. е. функционал действует по переменной $y$.

Теперь несложные операции с носителями этих сверток убеждают нас в том, что

$$
\operatorname{supp}\left(\omega_{+} * u_{+}^{(\varepsilon)}\right) \subset \bar{C}_{+}^{a}, \quad \operatorname{supp}\left(\omega_{-}^{-1} * u_{-}^{(\varepsilon)}\right) \subset \mathbb{R}^{2} \backslash C_{+}^{a} .
$$

Предельньй переход при $\varepsilon \rightarrow 0$ показьвает, что

$$
\operatorname{supp}\left(A_{\neq} u_{+}\right)(x) \subset \partial \bar{C}_{+}^{a}, \quad \operatorname{supp}\left(A_{=}^{-1} u_{-}\right)(x) \subset \mathbb{R}^{2} \backslash C_{+}^{a},
$$


а применение преобразования Фурье уже дает

$$
A_{\neq}(\xi) \widetilde{u}_{+}(\xi) \in \widetilde{H}_{s-\varkappa}\left(C_{+}^{a}\right), \quad A_{=}^{-1}(\xi) \widetilde{u}_{-}(\xi) \in \widetilde{H}_{s-\varkappa}\left(\mathbb{R}^{2} \backslash \bar{C}_{+}^{a}\right) .
$$

Из (25) по теореме 5 (так как $|s-\varkappa|<1 / 2)$ имеем $A_{\neq}(\xi) \widetilde{u}_{+}(\xi)=G_{2} A_{=}^{-1}(\xi) \tilde{l f}(\xi)$ и, следовательно, $\widetilde{u}_{+}(\xi)=A_{\neq}^{-1}(\xi) G_{2} A_{=}^{-1}(\xi) \widetilde{l f}(\xi)$, причем найденное решение единственно. Априорная оценка получается ввиду ограниченности операторов $A_{\neq}^{-1}, G_{2}, A_{=}^{-1}$ в соответствующих пространствах:

$$
\begin{aligned}
\left\|u_{+}\right\|_{s} & =\left\|\widetilde{u}_{+}\right\|_{s} \leqslant c\left\|G_{2} A_{=}^{-1}(\xi) \tilde{l f}(\xi)\right\|_{s-\varkappa} \\
& \leqslant c\left\|A_{=}^{-1}(\xi) \widetilde{l f}(\xi)\right\|_{s-\varkappa} \leqslant c\|\widetilde{l f}\|_{s-\alpha}=c\|l f\|_{s-\alpha} \leqslant c\|f\|_{s-\alpha}^{+} .
\end{aligned}
$$

Стоит отметить, что решение уравнения (19) не зависит от выбора продолжения $l f-$ следствие аналитичности символов $A_{\neq}, A_{=}$и проекционных свойств оператора $G_{2}$.

8. Подобная факторизация уже встречалась в работе [12] при решении некоторой многомерной задачи линейного сопряжения, обобщающей известную краевую задачу Римана [11].

Выбор термина “волновая факторизация” обусловлен свойствами так называемых причинных функций и их связью с волновым уравнением [13].

\section{СПИСОК ЦИТИРОВАННОЙ ЛИТЕРАТУРЫ}

[1] Вишик М. И., Эскин Г. И. Уравнения в свертках в ограниченной области // УМН. 1965. T. 20. № 3. C. 89-152.

[2] Эскин Г.И. Краевые задачи для эллиптических псевдодифференциальных уравнений. М.: Наука, 1973.

[3] Boutet de Monvel L. Boundary problems for pseudo-differential equations // Acta Math. 1971. V. 126. № 1-2. P. 11-51.

[4] Ремпель Ш., Шульце Б.-В. Теория индекса эллиптических краевых задач. М.: Мир, 1986.

[5] Кондратьев В.А., Олейник О.А. Краевые задачи для уравнений с частными производными в негладких областях // УМН. 1983. Т. 38. № 2. С. 3-76.

[6] Владимиров В.С. Методы теории функций многих комплексных переменных. М.: Наука, 1964.

[7] Васильев В. Б. Сингулярные интегралы на компактных многообразиях с особенностями // Дифференц. уравнения. 1993. Т. 29. № 9. С. 1642-1643.

[8] Mikhlin S. G., Prössdorf S. Singular Integral Operators. Berlin: Academie-Verlag, 1986.

[9] Мальгранж Б. Сингулярные интегральные операторы и единственность задачи Коши // Математика. Сб. переводов. 1962. Т. 6. № 5. С. 87-129.

[10] Владимиров В. С. Обобщенные функции в математической физике. М.: Наука, 1979.

[11] Гахов Ф. Д. Краевые задачи. М.: Наука, 1977.

[12] Владимиров В. С. Задача линейного сопряжения для голоморфных функций // Изв. АН CССР. Сер. матем. 1965. Т. 29. № 4. С. 807-834.

[13] Паламодов В. П. Обобщенные функции и гармонический анализ // Современные проблемы математики. Фундаментальные направления. Итоги науки и техники. Т. 72. М.: ВИНИТИ, 1991. C. $5-134$. 